\title{
Associations among gastroesophageal reflux disease, mental disorders, sleep and chronic temporomandibular disorder: a case-control study
}

\author{
Yuanyuan Li DDS MS, Ming Fang DDS PhD, Lina Niu DDS PhD, Yu Fan DDS MS, Yan Liu DDS MS, Yong Long PhD, \\ Xiaodong Liu DDS PhD, Franklin R. Tay BDSc PhD, Jihua Chen DDS PhD
}

Cite as: CMAJ 2019 August 19;191:E909-15. doi: 10.1503/cmaj.181535

\begin{abstract}
BACKGROUND: Temporomandibular disorders (TMDs) are a family of pain-related disorders associated with impaired function in the jaw, temporomandibular joint and muscles of mastication. Our objectives were to evaluate the association between chronic TMD and gastresophageal reflux disease (GERD) and to determine whether mental disorders or undermined sleep mediates this association.
\end{abstract}

METHODS: We conducted a case-control study involving 1522 consecutive adult patients with chronic TMD and 1522 matched controls from 2 hospitals in China. All participants were aged between 18 and 70 years and were recruited from July 2017 to April 2018 Chronic TMD was diagnosed by trained dentists using the criteria in the Orofacial Pain Prospective Evaluation and Risk Assessment Study. Trained gastroenterologists made blinded diagnoses of GERD according to the Montreal definition and classification (at least $2 \mathrm{~d}$ of mild symptoms, or $1 \mathrm{~d}$ of moderate or severe symptoms per week). We used validated questionnaires to evaluate psychological status and sleep quality.

RESULTS: Of the study participants, we identified 132 patients and 61 controls with GERD. Using conditional logistic regression analysis, we identified GERD as a risk factor for TMD (odds ratio 2.74, 95\% confidence interval 1.88 to 3.98 ). Mediation analyses identified that somatization, anxiety and undermined sleep moderately mediated the relation between TMD and GERD.

INTERPRETATION: Our study suggests that symptomatic GERD is associated with chronic, painful TMD, and somatization, anxiety and undermined sleep mediate this association to a certain extent. Due consideration should be given to the evaluation and management of gastrointestinal symptoms and mental disorders in the combined therapy for painful TMD.
$\mathrm{T}$ emporomandibular disorders (TMDs) are a set of craniofacial pain-related disorders associated with impaired function in the jaw, temporomandibular joint and muscles of mastication. ${ }^{1}$ A 2015 clinical review found that pain was most frequently reported in both the muscles of mastication and the temporomandibular joint (myalgia with arthralgia in $73 \%$ of cases), followed by myalgia alone ( $23 \%$ of cases). ${ }^{2}$ Pain associated with TMD is found in $13 \%$ of the Canadian population. ${ }^{3}$ Like other musculoskeletal disorders including chronic low back pain, fibromyalgia and headache, chronic painful TMD cannot be explained by physical conditions. The cause of TMD has changed substantially from a mechanistic origin to one that is multifactorial and biopsychosocial in nature..$^{1,4}$

Chronic TMD has been associated with somatization, anxiety, depression, parafunctional behaviours, jaw injury and other chronic pain syndromes. ${ }^{1,2}$ Some authors have previously suggested a potential association between TMD and gastroesophageal reflux disease (GERD). ${ }^{5}$ Gastroesophageal reflux disease is diagnosed when reflux of stomach contents precipitates troublesome symptoms. ${ }^{6}$

Sufficient evidence supports the relation between GERD and mental disorders including somatization, anxiety and depression. ${ }^{7-9}$ Psychological factors and somatic symptoms are closely associated with onset of TMD and persistence. ${ }^{10}$ Mental disorders are either potential mediators connecting GERD and TMD or comorbidities caused by shared pathophysiological processes. ${ }^{5}$ Undermined sleep mediates the effect of stress on painful TMD. ${ }^{11}$ Considering the association between GERD and undermined sleep,${ }^{9}$ it is possible that undermined sleep is a mediator linking GERD with TMD. Current evidence of an association between TMD and GERD from a case-control study provides only descriptive 
findings without estimation of the strength of association and consideration of confounding factors, apart from the limited number of participants in the study. ${ }^{5}$

The aims of this study were to evaluate the relation between chronic TMD and GERD in a larger group of patients, to evaluate the strength of association after adjusting for potential confounding factors and to assess whether mental disorders or undermined sleep mediates this association. The null hypotheses examined were that there is no relation between chronic TMD and GERD; and that somatization, anxiety, depression and undermined sleep do not mediate the association between TMD and GERD.

\section{Methods}

Reporting of this study defers to the checklist of Strengthening the Reporting of Observational Studies in Epidemiology (STROBE) statement: guidelines for reporting observational studies. ${ }^{12}$

\section{Study design and participants}

We conducted a case-control study involving patients aged 18-70 years who were diagnosed consecutively with chronic TMD and controls without chronic TMD. We recruited patients and controls from the Stomatological Hospital of the Fourth Military Medical University, Xi'an, Shaanxi, China (academic hospital in an urban setting) and the 188th Hospital of the Chinese People's Liberation Army, Chaozhou, Guangdong, China (nonacademic hospital in an urban setting) (details in Appendix 1, available at www.cmaj.ca/lookup/suppl/doi:10.1503/cmaj.181535/-/DC1). We enrolled participants from July 2017 to April 2018. Each participant signed an informed consent form.

Patients, including those who were referred for care for orofacial pain, were examined by 2 dentists ( 1 from each study site). The dentists were trained and calibrated at the Stomatological Hospital of the Fourth Military Medical University ahead of participant enrollment (Appendix 1). Chronic TMD was diagnosed using criteria from the Orofacial Pain Prospective Evaluation and Risk Assessment Study (OPPERA). ${ }^{4}$ Briefly, the inclusion criteria were frequently reported pain in the face, muscles of mastication, temples or temporomandibular joints in the last 6 months (more than 14 days in the last month and more than 4 days/month in the previous 5 months); pain in the facial regions defined by the dentists for more than 4 days during the past month; or pain in at least 3 muscles of mastication or at least 1 temporomandibular joint during maneuvering of the mandible or palpation of the muscles. ${ }^{4}$ The facial regions were defined by the dentists via palpating the temporalis, the preauricular region, the masseter muscle, the posterior mandibular region and the submandibular region bilaterally. ${ }^{4}$

We recruited control participants from patients seeking consultation for other dental diseases in the same hospital. One control participant was matched to each TMD case by age (within $2 \mathrm{yr}$ ) and sex. We randomly selected potential control participants using SPSS 22.0 software from a list that included age, sex, identification number and mobile phone number of patients who registered on the same day as the participants diagnosed with TMD. Potential participants were invited and were examined by the study dentists to determine if they were qualified for the study. Inclusion criteria for the control participants were no orofacial pain during the last month and occurring fewer than 5 days/month in the previous 5 months; headaches occurring fewer than 5 days/month in the previous 3 months; pain in the dentist-defined orofacial regions occurring fewer than 5 days in the last month; diagnosed with no myalgia or arthralgia; no previous diagnosis of TMD; and no use of occlusal splint. ${ }^{4}$ Patients seeking general dental treatment for tooth erosion were excluded from the control cohort. Details of participant selection are described in Appendix 1.

\section{Data collection}

The 2 study dentists collected data on personal characteristics and potential confounding factors during the face-to-face interviews with the study participants. We collected data for age, sex, place of residence, education, marital status, height, weight, medical history, and consumption of cigarettes, alcohol, tea and coffee. We evaluated smoking status using questions proposed by the World Health Organization. ${ }^{13}$ Amount of alcohol consumption in the past year was asked and recorded. Participants were identified as nondrinkers, moderate drinkers or heavy drinkers based on the Chinese Dietary Guidelines. ${ }^{14}$ The frequency of consuming 1 cup of coffee or tea was asked and recorded according to the following categories (never or hardly ever, $<1$ time/wk, 1-2 times/wk, 3-6 times/wk, 1 time/d, 2-3 times/d, 4-5 times/d and $\geq 6$ times/d). ${ }^{15}$ We previously published the procedure for data collection. ${ }^{15}$

We also obtained information on oral behaviours involving the jaw over the previous month during the interview by asking 8 questions on the following items: gum chewing, biting hard objects, leaning with the hand on the jaw, cheek biting, chewing food on 1 side, clenching/grinding teeth during waking hours, clenching/grinding teeth when asleep and sleeping on the side. ${ }^{16} \mathrm{~A}$ dichotomous answer (yes/no) was required for each question, with "yes" indicating monthly or more frequently and "no" indicating less than monthly. The frequency of each oral behaviour was also noted. Those who were uncertain of the information were designated as "no."

We employed validated questionnaires to identify patients with somatization, depression, anxiety and undermined sleep. All questionnaires were scored immediately after they were completed by the patient. A score of 10 or more on the 15 -item Patient Health Questionnaire (PHQ-15), ${ }^{17}$ the Generalized Anxiety Disorder 7-item (GAD-7) scale ${ }^{18}$ and the 9-item Patient Health Questionnaire (PHQ-9) ${ }^{19}$ were used to identify patients with somatization, anxiety and depression, respectively. We used a score of 6 or more on the Pittsburgh Sleep Quality Index (PSQI) to identify patients with undermined sleep. ${ }^{20}$ For the PHQ-15, we allowed as many as 5 missing items for the score to be considered valid; for example, if 5 items were missing, then the remaining 10 items were summed up, divided by 10 , and multiplied by 15 to generate a score as though all 15 items had valid scores. ${ }^{21}$ For the GAD-7, the PHQ-9 and the PSQI, we allowed as many as 2 missing items for the score to be considered valid. The Cronbach $\alpha$ values for PHQ-15, GAD-7, PHQ-9 and PSQI were 0.94, $0.90,0.86$ and 0.92 , respectively.

We used the GerdQ questionnaire to screen patients with GERD. ${ }^{22}$ Participants who reported regurgitation, heartburn, 
nausea, epigastric pain, additional medication because of reflux symptoms or disturbed sleep resulting from reflux symptoms during the previous week were further examined by 2 trained gastroenterologists (1 from each study site). The gastroenterologists made a blinded diagnosis of GERD according to the Montreal definition and classification: at least 2 days of mild symptoms or 1 day of moderate or severe symptoms per week. ${ }^{6}$ The duration and frequency of symptoms were noted. We recorded how long and how often the participants had reflux symptoms.

Research team members (dentists, gastroenterologists and other investigators) were selected based on their discipline and were trained to diagnose or administer questionnaires as per the study protocol (Appendix 1).

\section{Estimation of sample population size}

We conducted a pilot study involving 72 participants-with and 72 participants without chronic TMD that estimated an odds ratio (OR) of 2.5 for TMD related to GERD. If $3.8 \%$ of the population in China had GERD ${ }^{23}$ and 1 control was matched to 1 case, a sample size of 437 cases would provide $90 \%$ power $(\alpha 0.05) .^{24}$ Accordingly, we recruited 1522 cases for the present study to enable adjustment of confounding variables.

\section{Statistical analysis}

We performed Mann-Whitney $U$ tests to analyze continuous variables and $\chi^{2}$ tests to analyze categorical variables. We performed conditional logistic regression to evaluate the relation between TMD and GERD, conditioning on matched sets. We used multiple imputation to replace missing data (Appendix 2, available at www. cmaj.ca/lookup/suppl/doi:10.1503/cmaj.181535/-/DC1). Multivariate analyses were adjusted for demographic characteristics (Appendix 2). Teeth clenching or grinding during sleep and during waking hours were also included as potential confounders in regression analyses because these 2 factors were associated with TMD in the univariate analyses and associated with GERD in previous research. ${ }^{15}$ We conducted sensitivity analyses using complete cases. We used goodness-of-fit tests to evaluate the calibration of every regression model.

Because GERD increases the likelihood of subsequent mental disorders and sleep disorders, ${ }^{9}$ and the latter contribute to the development of TMD, ${ }^{4,10}$ we assumed that mental disorders and undermined sleep were mediators for the association between GERD and TMD. Accordingly, we used mediation analysis to test the hypothesis that an antecedent variable $X$ exerts its effects on a resultant variable $Y$ via mediator variables. Mediation analysis may be conducted even if causality cannot be established because of the limitations of study design and data collection. ${ }^{25}$ The method of mediation analysis in a case-control study has been published elsewhere. ${ }^{26}$ The mediation proportions of somatization, anxiety, depression and undermined sleep on the association between GERD and TMD were identified by mediation analysis using the PROCESS Procedure, with 5000 bias-corrected bootstrap samples for confidence intervals. ${ }^{27}$ To examine the mediation proportions of somatization, anxiety, depression and undermined sleep, we analyzed the scores of the questionnaires as continuous parameters. Details of mediation analyses are described in Appendix 2.
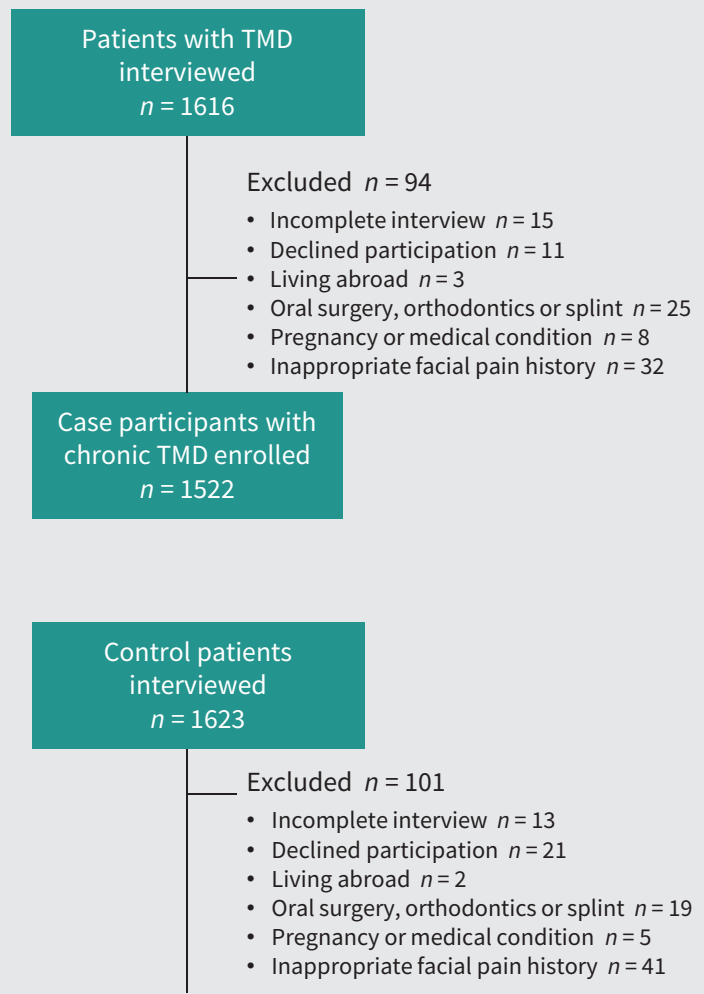

Control participants

enrolled

$n=1522$

Figure 1: Flow charts for study enrollment of case participants and control participants. Note: $\mathrm{TMD}=$ temporomandibular disorder.

We used SPSS 22.0, PROCESS v2.16 and R 2.15 software for all analyses (2-sided $\alpha$ value 0.05 ).

\section{Ethics approval}

This study was approved by the Ethics committees of the Stomatological Hospital of the Fourth Military Medical University and the 188th Hospital of the Chinese People's Liberation Army.

\section{Results}

We initially considered 1616 consecutive patients with TMD and ultimately enrolled 1522 case participants (94.2\%) with chronic TMD. Figure 1 shows the participant enrollment process for the study. We initially invited 1623 control participants and subsequently enrolled 1522 (93.8\%).

Table 1 provides the main characteristics of the 3044 participants. Of all participants, women accounted for $68.9 \%$ (1048/1522). Among all participants with TMD, 487 (32.0\%) had myalgia (pain in the muscles of mastication) alone, 181 (11.9\%) had arthralgia (pain in the temporomandibular joint) alone, and the remaining $(854,56.1 \%)$ had both arthralgia and myalgia. Among the 201 participants with GERD, 31 case participants and 12 control participants were taking antacid medications before the start of the study. Twelve participants $(5$ case and 7 control 
participants) did not know the duration of their reflux symptoms and we considered these patients to have a 5-year or shorter duration of symptoms. Information from the interviews was complete except for data about consumption of cigarettes by current smokers and body mass index (BMI; Supplementary
Table S1, Appendix 3, available at www.cmaj.ca/lookup/suppl/ doi:10.1503/cmaj.181535/-/DC1). Because the completeness of all the questionnaires was examined by the dentists immediately following patient completion, there were no missing data on the questionnaires.

Table 1: Characteristics of study participants

\section{Characteristic}

Age, median (IQR); yr

Sex

Male

Female

Residence

Urban

Rural

BMI, median (IQR); kg/m²

BMI; $\mathrm{kg} / \mathrm{m}^{2}$

Underweight or normal weight $(<25)$

Overweight $(25$ to $<30)$

Obese $(\geq 30)$

Marital status

Never married

Married

Divorced or widowed

Education level (yr)

$\leq 9$

$>9-12$

$>12$

Smoking status

Never

Former

Current

Cigarettes smoked, median (IQR); no. cigarettes/d†

Alcohol consumption

None or moderate

Heavy

Tea consumption

None or less than weekly

Weekly

Daily

Coffee consumption

None or less than weekly

Weekly

Daily
No. (\%) of participants with TMD (cases) $n=1522^{\star}$

$27(22-38)$

474 (31.1)

$1048(68.9)$

$1192(78.3)$

330 (21.7)

$20.8(18.8-22.9)$

1358 (89.9)

138 (9.1)

$15(1.0)$

785 (51.6)

$709(46.6)$

$28(1.8)$

140 (9.2)

257 (16.9)

$1125(73.9)$

$1291(84.8)$

$61(4.0)$

170 (11.2)

$10(5-20)$

$1320(86.7)$

202 (13.3)

$983(64.6)$

367 (24.1)

$172(11.3)$

1311 (86.1)

179 (11.8)

$32(2.1)$
No. (\%) of participants without TMD (controls) $n=1522^{\star}$

$p$ value

$28(23-39)$

0.06

$>0.99$

474 (31.1)

$1048(68.9)$

0.45

$1209(79.4)$

$313(20.6)$

$21.4(19.2-23.7)$

$<0.001$

$<0.001$

$1283(85.1)$

207 (13.7)

$18(1.2)$

745 (48.9)

757 (49.7)

$20(1.3)$

0.4

$158(10.4)$

$271(17.8)$

$1093(71.8)$

$1303(85.6)$

$64(4.2)$

$155(10.2)$

$10(6-20)$

$1327(87.2)$

$195(12.8)$

849 (55.8)

472 (31.0)

$201(13.2)$

0.003

$1243(81.7)$

$241(15.8)$

$38(2.5)$

Note: $B M I=$ body mass index, $I Q R=$ interquartile range, $T M D=$ temporomandibular disorder

*Unless stated otherwise.

tConsumption by current smokers. 
With adjustment of potential confounders, we identified symptomatic GERD as a risk factor for TMD (Table 2). We found that participants with a longer history of GERD symptoms had a slightly higher OR for TMD than those with a shorter duration (Table 2). Sensitivity analyses showed similar results (Supplementary Table S2, Appendix 3). Observed and expected data matched well in the goodness-of-fit tests.

Mediation analyses supported that somatization, anxiety and undermined sleep were mediators in the association between TMD and GERD (Table 3). Somatization, anxiety and undermined sleep mediated $14 \%, 11 \%$ and $10 \%$ of the association between TMD and GERD, respectively (Figure 2). Depression was not a mediator of the association.

\section{Interpretation}

We found that chronic TMD is associated with symptomatic GERD. Hence, we rejected our first null hypothesis. Somatization, anxiety and undermined sleep but not depression are significant mediators in the association between TMD and GERD. Hence, our second null hypothesis can only be partially rejected.

Although there was evidence from a previous case-control study linking TMD and GERD, these findings were descriptive only. ${ }^{5}$ By evaluating the strength of association and showing potential mediators in the pathway, our findings add key knowledge to the literature and should prompt further research on the mechanisms.

We note that the positive mediation associations reported in our study are consistent with but could not prove causation. The interactions between chronic musculoskeletal diseases, gastrointestinal diseases, mental disorders and sleep problems are complicated. There is evidence to support the bidirectional nature of the associations among these comorbidities, ${ }^{28}$ and patients may be stuck in a cycle in which undermined sleep, somatization and anxiety exacerbate the pain, with the pain also leading to sleep problems and mental disorders. ${ }^{28}$ We found no association between depression and TMD, which is contrary to the studies involving populations in Western countries, ${ }^{10,29}$ but is in line with studies involving populations in Asia., ${ }^{50}$ This finding may be explained partially by the more reserved nature of Asian populations when responding to questions about depression compared with Western populations. ${ }^{5}$ The associations among mental disorders, GERD and TMD may also be underestimated for the same reason.

The finding that the mediation association does not make up $100 \%$ of the whole association suggests the existence of other mechanisms linking GERD with TMD. The association between

Table 2: Association between gastroesophageal reflux disease and chronic

temporomandibular disorder using conditional logistic regression, with missing data imputed by multiple imputation

\begin{tabular}{|c|c|c|c|c|}
\hline Variable & $\begin{array}{c}\text { No. }(\%) \text { of } \\
\text { participants } \\
\text { with TMD } \\
\text { (cases) } \\
n=1522^{\star}\end{array}$ & $\begin{array}{l}\text { No. }(\%) \text { of } \\
\text { participants } \\
\text { without } \\
\text { TMD } \\
\text { (controls) } \\
n=1522^{\star}\end{array}$ & $\begin{array}{l}\text { Univariable } \\
\text { analysis }\end{array}$ & $\begin{array}{c}\text { Multivariable } \\
\text { analysis } †\end{array}$ \\
\hline GERD & $132(8.7)$ & $61(3.5)$ & 2.61 (1.88 to 3.63 ) & 2.74 (1.88 to 3.98 ) \\
\hline GERD, duration $\leq 5 \mathrm{yr}$ & $61(4.0)$ & $25(1.6)$ & $2.60(1.62$ to 4.19$)$ & 2.53 (1.47 to 4.35$)$ \\
\hline GERD, duration $>5 \mathrm{yr}$ & $71(4.7)$ & $28(1.8)$ & 2.62 (1.69 to 4.07 ) & 2.90 (1.80 to 4.69$)$ \\
\hline
\end{tabular}

Table 3: Mediation analysis* of the association between gastroesophageal reflux disease and temporomandibular disorder

\begin{tabular}{|c|c|c|c|c|c|}
\hline Mediator & $\begin{array}{l}\text { No. }(\%) \text { of } \\
\text { participants } \\
\text { with TMD } \\
\text { (cases) } \\
n=1522\end{array}$ & $\begin{array}{c}\text { No. }(\%) \text { of } \\
\text { participants } \\
\text { without TMD } \\
\text { (controls) } \\
n=1522\end{array}$ & $\begin{array}{c}\text { Indirect effect } \\
(95 \% \mathrm{Cl})\end{array}$ & $\begin{array}{c}\text { Direct effect } \\
(95 \% \mathrm{CI})\end{array}$ & $\begin{array}{c}\text { Coefficient } \\
(95 \% \mathrm{Cl})\end{array}$ \\
\hline Somatization & $549(36.1)$ & $301(19.8)$ & $0.13(0.07$ to 0.20$)$ & $0.79(0.44$ to 1.13$)$ & 0.059 (0.045 to 0.072 ) \\
\hline Anxiety & $135(8.9)$ & $80(5.3)$ & $0.10(0.06$ to 0.17$)$ & 0.81 (0.46 to 1.15 ) & 0.052 (0.031 to 0.073$)$ \\
\hline Depression & $217(14.3)$ & $188(12.4)$ & $0.03(-0.01$ to 0.08$)$ & 0.86 (0.52 to 1.21$)$ & 0.014 (-0.003 to 0.031$)$ \\
\hline Undermined sleep & $663(43.6)$ & $535(35.2)$ & 0.09 (0.05 to 0.15$)$ & 0.81 (0.47 to 1.16$)$ & $0.047(0.025$ to 0.070$)$ \\
\hline
\end{tabular}

Note: $\mathrm{Cl}=$ confidence interval, $\mathrm{TMD}=$ temporomandibular disorder.

*Adjusted for age, sex, residence, body mass index, education, marital status, smoking status, alcohol consumption, tea consumption, coffee consumption, teeth clenching or grinding during sleep and teeth clenching or grinding during waking hours. 


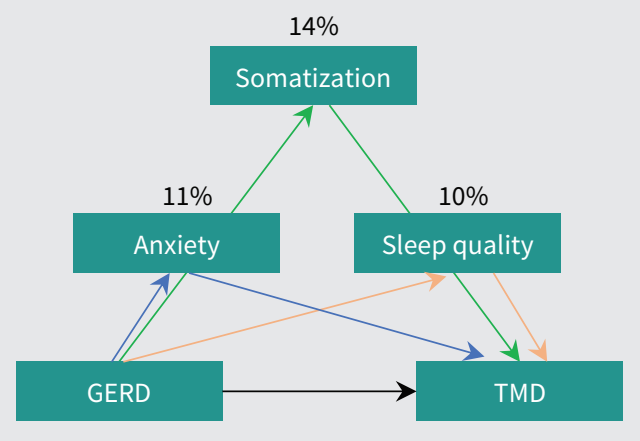

Figure 2: Mediation models for the association between temporomandibular disorder (TMD) and gastrosophageal reflux disease (GERD). Somatization, anxiety and undermined sleep mediated $14 \%, 11 \%$ and $10 \%$ of the association between TMD and GERD, respectively. Arrows represent the possible direct and indirect effects of mediation.

GERD and teeth grinding or clenching has been reported previously. ${ }^{15,31-34}$ Esophageal acidification not only increases rhythmic masticatory muscle activity, along with teeth clenching or grinding during sleep, ${ }^{35}$ but also increases masseter muscle activities during waking hours. ${ }^{36}$ Muscular pain in patients with TMD could be associated with muscle fatigue caused by these GERD-related oral behaviours. ${ }^{5}$

We did not design our study to evaluate the temporal associations among these conditions; however, this issue is still intriguing. Further longitudinal studies may advance knowledge of the underlying mechanisms. For example, esophageal acidification may sensitize the central nervous system to sensory and pain pathways, resulting in declined pain thresholds in the other organs. Temporomandibular disorders have been associated with gastrointestinal disorders, especially irritable bowel syndrome (IBS). ${ }^{2,37}$ Comorbidity of GERD and IBS is common in the general population. ${ }^{38}$ Although we did not evaluate these comorbidities systematically, the finding of an association between GERD and TMD is consistent with the literature. Future studies evaluating such comorbidities should be informative.

Chronic pain is a considerable stress on the Canadian economy, with an estimated annual economic cost of about 43 billion dollars in health care and lost productivity. ${ }^{39}$ The Canadian Pain Task Force was established in 2019 to advance solutions for the management of chronic pain. ${ }^{40}$ Recent evidence supports the effectiveness and cost-effectiveness of multidisciplinary pain management with psychotherapies. ${ }^{41}$ Recent clinical guidelines highlight the importance of brain-gut psychotherapies and multidisciplinary rehabilitation in the management of chronic pain ${ }^{42}$ and chronic gastrointestinal diseases, including GERD and IBS. ${ }^{43}$

\section{Limitations}

We completed 1:1 matching between case and control participants, with matching on age and sex. Several principles have been developed in the selection of control participants for casecontrol studies. ${ }^{44,45}$ Adherence to these principles can reduce bias. However, it is difficult to achieve full compliance with these principles just as perfect experimental conditions are unlikely to be achieved in a laboratory. Therefore, tolerance of a small deviation from the principles is usually the norm for investigating exposure-disease associations. ${ }^{44}$ Although the matching protocol may not be perfect, useful information can still be generated from our analyses.

Because participants were recruited from hospitals, selection bias is a concern, and the results may not represent the general population conclusively. To enhance the generalizability of the findings, we recruited participants from 2 hospitals at disparate levels (academic tertiary hospital v. nonacademic secondary hospital) from diverse areas of China. If exposure extents were different between hospital controls and the population that generated the cases, selection bias would occur. ${ }^{46}$ Therefore, we excluded patients seeking treatment for tooth erosion related to GERD from the control cohort. ${ }^{45}$ The large patient cohort also contributed to the reduction of selection bias.

A cross-sectional study published in 2016 found that the prevalence of GERD in the adult population in China was 3.8\%. ${ }^{23}$ When we applied an indirect standardization of rates, the standardized proportion of patients with GERD (4.0\%) among the 3044 participants was close to the reported prevalence in the Chinese population. Some participants failed to provide data on BMI because they could not recall their weight or height. Accordingly, we conducted multiple imputation under the assumption of "missing at random" (Appendix 2). The likelihood of bias is minimal because there was no distinct difference between the primary and sensitivity analyses.

Another limitation of our study is that a temporal relation cannot be established because of the study design. In addition, there may be shared risk factors and unmeasured confounders that we did not evaluate in the present study.

\section{Conclusion}

We found that symptomatic GERD is associated with painful chronic TMD, and somatization, anxiety and undermined sleep mediate this association moderately. Physicians and patients may overlook the association between chronic musculoskeletal diseases and gastrointestinal symptoms. Patients with both chronic TMD and reflux symptoms may be underdiagnosed, resulting in deferred effective treatment and a prolonged disease course.

\section{References}

1. Scrivani SJ, Keith DA, Kaban LB. Temporomandibular disorders. N Engl J Med 2008;359:2693-705.

2. Durham J, Newton-John TR, Zakrzewska JM. Temporomandibular disorders. BMJ 2015;350:h1154.

3. Locker D, Slade G. Prevalence of symptoms associated with temporomandibular disorders in a Canadian population. Community Dent Oral Epidemiol 1988; 16:310-3.

4. Slade GD, Ohrbach R, Greenspan JD, et al. Painful temporomandibular disorder: decade of discovery from OPPERA studies. J Dent Res 2016;95:1084-92.

5. Gharaibeh TM, Jadallah K, Jadayel FA. Prevalence of temporomandibular disorders in patients with gastroesophageal reflux disease: a case-controlled study. J Oral Maxillofac Surg 2010;68:1560-4.

6. Vakil N, van Zanten SV, Kahrilas P, et al. The Montreal definition and classification of gastroesophageal reflux disease: a global evidence-based consensus. Am J Gastroenterol 2006;101:1900-20; quiz 43. 
7. Baker LH, Lieberman D, Oehlke M. Psychological distress in patients with gastroesophageal reflux disease. Am J Gastroenterol 1995;90:1797-803.

8. You ZH, Perng CL, Hu LY, et al. Risk of psychiatric disorders following gastroesophageal reflux disease: a nationwide population-based cohort study. Eur J Intern Med 2015;26:534-9.

9. On ZX, Grant J, Shi Z. The association between gastroesophageal reflux disease with sleep quality, depression, and anxiety in a cohort study of Australian men. J Gastroenterol Hepatol 2017;32:1170-7.

10. Fillingim RB, Ohrbach R, Greenspan JD, et al. Psychological factors associated with development of TMD: the OPPERA prospective cohort study. J Pain 2013;14:T75-90.

11. Sanders AE, Akinkugbe AA, Fillingim RB, et al. Causal mediation in the development of painful temporomandibular disorder. J Pain 2017;18:428-36.

12. von Elm E, Altman DG, Egger M, et al. The Strengthening the Reporting of Observational Studies in Epidemiology (STROBE) statement: guidelines for reporting observational studies. Lancet 2007;370:1453-7.

13. Guidelines for controlling and monitoring the tobacco epidemic. Geneva: World Health Organization; 1998.

14. The Chinese dietary guidelines. Beijing: Chinese Nutrition Society; updated 2016 Sept. 30. Available: http://dg.cnsoc.org/index.html (accessed 2018 June 13).

15. Li Y, Yu F, Niu L, et al. Association between bruxism and symptomatic gastroesophageal reflux disease: a case-control study. J Dent 2018;77:51-8.

16. Akhter R, Hassan NM, Ohkubo R, et al. The relationship between jaw injury, third molar removal, and orthodontic treatment and TMD symptoms in university students in Japan. J Orofac Pain 2008;22:50-6.

17. Kroenke K, Spitzer RL, Williams JB. The PHQ-15: validity of a new measure for evaluating the severity of somatic symptoms. Psychosom Med 2002;64:258-66.

18. Spitzer RL, Kroenke K, Williams JB, et al. A brief measure for assessing generalized anxiety disorder: the GAD-7. Arch Intern Med 2006;166:1092-7.

19. Kroenke K, Spitzer RL, Williams JB. The PHQ-9: validity of a brief depression severity measure. J Gen Intern Med 2001;16:606-13.

20. Buysse DJ, Reynolds CF III, Monk TH, et al. The Pittsburgh Sleep Quality Index: a new instrument for psychiatric practice and research. Psychiatry Res 1989;28:193-213.

21. Ohrbach R, Knibbe W. Diagnostic criteria for temporomandibular disorders: scoring manual for self-report instruments. Alexandria (VA): International Association for Dental Research (IADR); updated 2016 Nov. 6. Available: www.iadr. org/Portals/69/docs/Groups/INfORM/DC-TMD-Self-report-Instrument-Scoring -Manual_2017_01_09.pdf (accessed 2019 July 31).

22. Jonasson C, Wernersson B, Hoff DA, et al. Validation of the GerdQ questionnaire for the diagnosis of gastro-oesophageal reflux disease. Aliment Pharmacol Ther 2013;37:564-72.

23. Tan VP, Wong BC, Wong WM, et al. Gastroesophageal reflux disease: crosssectional study demonstrating rising prevalence in a Chinese population. J Clin Gastroenterol 2016;50:e1-7.

24. Machin D, Campbell MJ, Tan SB, et al. Sample size tables for clinical studies. 3rd edition. Chichester (UK): Wiley-Blackwell; 2009.

25. Hayes AF. Introduction to mediation, moderation, and conditional process analysis: a regression-based approach. 2nd ed. New York: Guilford Press; 2018.

26. VanderWeele TJ, Tchetgen Tchetgen EJ. Mediation analysis with matched case-control study designs. Am J Epidemiol 2016;183:869-70.
27. Preacher KJ, Hayes AF. Asymptotic and resampling strategies for assessing and comparing indirect effects in multiple mediator models. Behav Res Meth ods 2008;40:879-91.

28. Dieppe P. Chronic musculoskeletal pain. BMJ 2013;346:f3146.

29. Kim SE, Chang L. Overlap between functional GI disorders and other functional syndromes: What are the underlying mechanisms? Neurogastroenterol Motil 2012;24:895-913.

30. Yap AU, Dworkin SF, Chua EK, et al. Prevalence of temporomandibular disorder subtypes, psychologic distress, and psychosocial dysfunction in Asian patients. J Orofac Pain 2003;17:21-8.

31. Li Y, Yu F, Niu L, et al. Associations among bruxism, gastroesophageal reflux disease, and tooth wear. J Clin Med 2018;7:E417.

32. Mengatto CM, Dalberto Cda S, Scheeren B, et al. Association between sleep bruxism and gastroesophageal reflux disease. J Prosthet Dent 2013;110:349-55.

33. Ohmure H, Kanematsu-Hashimoto K, Nagayama K, et al. Evaluation of a proton pump inhibitor for sleep bruxism: a randomized clinical trial. J Dent Res 2016;95:1479-86.

34. Watanabe M, Nakatani E, Yoshikawa H, et al. Oral soft tissue disorders are associated with gastroesophageal reflux disease: retrospective study. $B M C$ Gastroenterol 2017;17:92.

35. Ohmure H, Oikawa K, Kanematsu K, et al. Influence of experimental esophageal acidification on sleep bruxism: a randomized trial. J Dent Res 2011;90:665-71.

36. Ohmure H, Sakoguchi Y, Nagayama K, et al. Influence of experimental oesophageal acidification on masseter muscle activity, cervicofacial behaviour and autonomic nervous activity in wakefulness. J Oral Rehabil 2014;41:423-31.

37. Whitehead WE, Palsson O, Jones KR. Systematic review of the comorbidity of irritable bowel syndrome with other disorders: What are the causes and implications? Gastroenterology 2002;122:1140-56.

38. Jung HK, Halder S, McNally M, et al. Overlap of gastro-oesophageal reflux disease and irritable bowel syndrome: prevalence and risk factors in the general population. Aliment Pharmacol Ther 2007;26:453-61.

39. Lynch ME. The need for a Canadian pain strategy. Pain Res Manag 2011; 16:77-80.

40. Canadian Pain Task Force. Ottawa: Government of Canada; updated 2019 July 23. Available: www.canada.ca/en/health-canada/corporate/about-health -canada/public-engagement/external-advisory-bodies/canadian-pain-task-force. html (accessed 2019 July 31).

41. Gatchel RJ, McGeary DD, McGeary CA, et al. Interdisciplinary chronic pain management: past, present, and future. Am Psychol 2014;69:119-30.

42. Gatchel RJ, Reuben DB, Dagenais S, et al. Research agenda for the prevention of pain and its impact: report of the work group on the prevention of acute and chronic pain of the Federal Pain Research Strategy. J Pain 2018;19:837-51.

43. Keefer L, Palsson OS, Pandolfino JE. Best practice update: incorporating psychogastroenterology into management of digestive disorders. Gastroenterology 2018;154:1249-57.

44. Wacholder S, McLaughlin JK, Silverman DT, et al. Selection of controls in casecontrol studies. I. Principles. Am J Epidemiol 1992;135:1019-28.

45. Grimes DA, Schulz KF. Compared to what? Finding controls for case-control studies. Lancet 2005;365:1429-33.

46. Galeotti F, Massari M, D'Alessandro R, et al. Risk of Guillain-Barré syndrome after 2010-2011 influenza vaccination. Eur J Epidemiol 2013;28:433-44.

\section{Competing interests: None declared.}

This article has been peer reviewed.

Affiliations: State Key Laboratory of Military Stomatology \& National Clinical Research Center for Oral Diseases \& Shaanxi Key Laboratory of Oral Diseases, Departments of Prosthodontics (Li, Fang, Niu, Fan, Y. Liu, Long, Tay, Chen), and Oral Anatomy and Physiology (X. Liu), School of Stomatology, The Fourth Military Medical University, Xi'an, Shaanxi, China; Department of Epidemiology, School of Public Health (Long), The Fourth Military Medical University, Xi'an, Shaanxi, China; Department of Endodontics (Tay, Chen), The Dental College of Georgia, Augusta University, Augusta, Ga.
Contributors: All of the authors participated in the design, implementation and interpretation of the study. Yuanyuan Li conducted the statistical analyses and had full access to the study data. Yuanyuan Li and Franklin Tay wrote the initial draft of the manuscript. All of the authors revised the manuscript critically for important intellectual content, gave final approval of the version to be published and agreed to be accountable for all aspects of the work.

Funding: This work was supported by grants from the National Key Research \& Development Program of China (grant nos. 2017YFC0840100 and 2017YFC0840109), National Natural Science Foundation of China (grant nos. 81720108011 and 81470773) and Changjiang Scholar Program of the Chinese Ministry of Education (grant no. IRT13051). The funding sources had no involvement in the design, conduct, or interpretation of the present work, or in the decision to submit the manuscript for publication.

Acknowledgments: The authors thank the clinical investigators at the Fourth Military Medical University and the 188th Hospital of the Chinese People's Liberation Army for their help with the present work.

Accepted: June 15, 2019

Correspondence to: Jihua Chen, jhchen@ fmmu.edu.cn; Franklin Tay, ftay@augusta. edu; Xiaodong Liu, xdliu@fmmu.edu.cn 